ROCZNIKI FILOZOFICZNE

Tom LXVIII, numer $4-2020$

DOI: https://doi.org/10.18290/rf20684-14

TOMASZ JARMUŻEK

KRZYSZTOF KRAWCZYK

\& RAFAŁ PALCZEWSKI

\title{
KNOWABILITY AS DE RE MODALITY: A CERTAIN SOLUTION TO FITCH PARADOX
}

\section{THE FITCH PARADOX - OVERVIEW}

Fitch paradox or Fitch-Church paradox, as it may also be called (see SALERNO 2009), is often considered as an argument against anti-realism. Accepting that every truth is knowable, as anti-realists do, leads to a paradoxical conclusion that there are no unknown truths, or equivalently, that every truth is in fact known. Yet, it is not 'the nail in the coffin' type of an argument, as series of solution proposals may suggest. In fact, there is some room to try to overcome the challenge. The main responses to the paradox based on either restricting the knowability principle: VAN BENTHEM 2004 and 2009; DuMMETT, 2001; Palczewski 2007; Tennant 1997 and 2009; or changing the logical base: Artemov and Protopopescu 2013; Beal 2000 and 2009; DeVidi and Solomon 2001; Kubyshikina and Zaitsev 2016; Maffezioli, Naibo, and Negri 2013; Priest 2009; Proietti 2012; Wansing 2002; Williamson 1982 and 1992 - more on different approaches, see BROGAARD and SALERno 2013.

First, we specify the language for the paradox. We have the set of propositional letters $\operatorname{Var}=\left\{p_{0}, p_{1}, \ldots\right\}$, classical connectives $\neg, \wedge, \vee, \rightarrow, \leftrightarrow$ and set of modal operators $\diamond, \square, K$, where the first two are alethic operators of possibility and necessity and the third is epistemic operator of knowledge.

TOMASZ JARMUŻEK, PhD Habil., Associate Professor at UMK (ORCID: https://orcid.org/0000-00033456-3859), Rafal Palczewski, PhD Habil., Associate Professor at UMK (ORCID: https://orcid.org/ 0000-0001-7368-8094), KrzyszTof KrawczyK, MA, PhD student (ORCID: https://orcid.org/ 00000003-4367-4796), Nicolaus Copernicus University in Torun (UMK), Faculty of Philosophy and Social Sciences, Department of Logic; address for correspondence: ul. Stanisława Moniuszki 16/20, 87-100 Toruń; e-mails: jarmuzek@umk.pl; rpalcz@umk.pl; krawczyk@doktorant.umk.pl. 
The resulting language will be called $\mathcal{L}$. The set of formulas is built in a standard way. We will call it $\operatorname{FOR}(\mathcal{L})$. Now the paradox is derivable in any logic that contains as theorems:

- all tautologies of Classical Propositional Logic, shortly: CPL,

- $K$ distribution over conjunction: $K(\varphi \wedge \psi) \rightarrow K \varphi \wedge K \psi\left(D i s_{K}^{\wedge}\right)$,

- Diamond-Box interdefinability: $\diamond \varphi \leftrightarrow \neg \square \neg \varphi(D B)$,

- Knowledge Factuality: $K \varphi \rightarrow \varphi(K F)$,

- Knowability Principle: $\varphi \rightarrow \diamond K \varphi(K P)$.

And rules:

- $\quad(M P) \frac{\varphi \rightarrow \psi, \varphi}{\psi}$

- $(G) \frac{\varphi}{\square \varphi}$.

The Hilbert style axiomatic system in which only axioms are all tautologies of CPL, $\left(D i s_{K}^{\wedge}\right),(D B),(K F),(K P)$ and rules are exactly $(M P)$ and $(G)$ will be called KFP - the kernel of Fitch paradox. The logic that is obtained within system KFP is the logic in which the paradox occurs.

Definition 1.1 (KFP-Proof). The KFP-proof of the formula $\varphi$ is the sequence $\psi_{1}, \ldots, \psi_{n}$, where $\psi_{n}=\varphi$, and for each $i \in\{1, \ldots, n\}, \psi_{i}$ fulfils at least one of the following:

- $\psi_{i}$ is an axiom of $\mathrm{KFP}$,

- there are $j, k<i$, such that $\psi_{j}=\psi_{k} \rightarrow \psi_{i}$,

- there is $j<i$, such that $\psi_{i}=\square \psi_{j}$,

We will write $\vdash_{\mathrm{KFP}} \varphi$ whenever there is a $\operatorname{KFP}$-proof of $\varphi$.

We also define the notion of deducibility:

Definition 1.2 Let $\Gamma \subseteq \operatorname{FOR}(\mathcal{L}), \varphi \in \operatorname{FOR}(\mathcal{L}) . \varphi$ is deducible from $\Gamma$ (shortly:

$\left.\Gamma \vdash_{\mathrm{KFP}} \varphi\right)$ iff there are $\psi_{1}, \ldots, \psi_{n} \in \Gamma$ such that $\vdash_{\mathrm{KFP}} \psi_{1} \wedge \ldots \wedge \psi_{n} \rightarrow \varphi$.

The proof of the paradoxical thesis may have the following form: 


$\begin{array}{lll}\text { (1) } \quad K(\varphi \wedge \neg K \varphi) \rightarrow(K \varphi \wedge K \neg K \varphi) & \left.\text { (Dis } s_{K}\right) \\ \text { (2) } & K \neg K \varphi \rightarrow \neg K \varphi & (K F) \\ \text { (3) } & K(\varphi \wedge \neg K \varphi) \rightarrow(K \varphi \wedge \neg K \varphi) & \text { (1), (2), (MP), CPL } \\ \text { (4) } & \neg(K \varphi \wedge \neg K \varphi) & \mathrm{CPL} \\ \text { Proof. } & \text { (5) } \neg K(\varphi \wedge \neg K \varphi) & \text { (3), (4), (MP), CPL } \\ & \text { (6) } \quad \square \neg K(\varphi \wedge \neg K \varphi) & \text { (5), (G) } \\ & \text { (7) } \neg \diamond K(\varphi \wedge \neg K \varphi) & \text { (6), (DB), (MP), CPL } \\ \text { (8) } & (\varphi \wedge \neg K \varphi) \rightarrow \diamond K(\varphi \wedge \neg K \varphi) & \text { (KP) } \\ \text { (9) } & \neg(\varphi \wedge \neg K \varphi) & \text { (7), (8), (MP), CPL } \\ \text { (10) } & \varphi \rightarrow K \varphi & \text { (9), (MP), CPL }\end{array}$

As we can see, in the proof we make use of formula $\varphi \wedge \neg K \varphi$ which is often called Moore's sentence. ${ }^{1}$ We obtain the 'unknowability' of such formula and then use it for a contraposition to finally get the paradoxical conclusion: $\varphi \rightarrow K \varphi$ which can be read in the following manner: if a sentence is true, then it is known.

We need to make some comments here. First of all, we made the stipulation that we need all formulas that are CPL tautologies in order for the paradox to occur. This is not true because we need only those tautologies that appear in the derivation itself. In this sense, KFP is not the minimal logic for the paradox. This idealisation is useful however, because we want our logic to be easy for semantical analysis. Having this in mind, we let KFP contain all CPL. Secondly, the lack of $K$ axiom and neccesitation rule for knowledge operator determines that our logic is not a normal modal logic. What is more, we do not need the equivalence in Diamond-Box interdefinability - implication is enough. However, if we wish to have some semantics behind the logic, it would be easier to extend the logic into the normal modal logic, since the semantics for those logics are best recognized. If we would like to obtain minimal normal modal logic for the paradox to

\footnotetext{
${ }^{1}$ We claim that it is incorrect, since 'Moore's sentence' means asserting both $p$ and $\neg K p-$ both assertions need to be made by one agent. Our opinion is that in the case of Fitch paradox, such a formula is stated from meta-level (for example by some objective observer about someone else's knowledge) and hence it is not paradoxical yet.
} 
occur, we would have to add $\square(\varphi \rightarrow \psi) \rightarrow(\square \varphi \rightarrow \square \psi)$ as an axiom, substitute $\left(D i s_{K}^{\wedge}\right)$ for $K(\varphi \rightarrow \psi) \rightarrow(K \varphi \rightarrow K \psi)$ and add the rule $\frac{\varphi}{K \varphi}$ to system $\mathrm{KFP}^{2}$. The second two modifications seem to be the most problematic since adding $\mathrm{K}$ axiom and neccesitation rule for knowledge results in so the called logical omniscience. It makes the whole setting highly idealised, but this is the price to pay if we wish to have simple and elegant semantics behind our logic.

\section{DE RE INTERPRETATION OF KNOWABILITY}

Knowability Principle $(K P)$ in the standard explication of the paradox (see FITCH 2009) is formalised by the composition of two modalitiespossibility $\diamond$ and knowledge $K$ :

$$
\varphi \rightarrow \diamond K \varphi
$$

We claim that what is problematic in Fitch Paradox is representing knowability as a composition of alethic operator of possibility and epistemic knowledge operator. It is at heart in the de dicto manner because the sentence $\diamond K p$ says that it is possible that agent knows that $p$. In possible world semantics it refers us to different accessible worlds similar to our world, from which at least one verifies that $K p$. Meanwhile, the knowability principle says that the truth of the sentence $p$ is enough for an agent to be able to know it in the world which is his actual world even if he is actually ignorant about $p$. Hence, we think that knowability principle should be interpreted as the one saying that truth of a sentence implies that an agent can know it in the initial world.

Also literally the sentence $\diamond K p$ says that the sentence $K p$ is possible. However, it is not the sentence $K p$ which is supposed to be possible, but an agent's knowledge about $p$. Let us look at the differences which are more apparent in the examples:

(a) It is possible that John knows that Torun is in Poland.

(b) John may know that Torun is in Poland.

In the case (a) we claim that a sentence is possible, whilst in case (b) we claim that a propositional attitude of knowledge is possible. It is a significant difference. Our distinction looks like, and in fact is, the distinction

\footnotetext{
${ }^{2}$ This approach is very much in the spirit of the one by Fischer (see FISCHER 2013, 64-76) who's motivation is — just as in our case — to analyse the paradox semantically.
} 
between de dicto and de re modalities. We claim that knowability principle is expressed in terms of de re modality. Hence, we do not treat knowability as a composition of modalities, but as a single modality. ${ }^{3}$ We substitute two modalities (alethic and epistemic one) by one modality expressing knowability. We will use the terms 'possible knowledge' and 'knowability' as denoting the same modality.

Before we examine the knowability logic, let us shortly mention former proposals that can be named "de re strategies in the context of Fitch paradox".

Zemach (1987) proposes to read formula $K \neg K p$ in the paradoxical argument as de re, not as de dicto. What does it mean in pracitce? Let $\mathrm{P}$ denote a given proposition (i.e. $\mathrm{P}$ is its name). Assume that John has forgotten what Pythagoras Theorem states, but he knows it is true. Then $K p$ interpreted as de dicto is not true, since John does not know the content of $\mathrm{P}$ that is expressed by the sentence substituted for $p$ (i.e. The square of the side opposite the right angle is equal to the sum of the squares of the other two sides). However, de re interpretation is true, because John knows that proposition $\mathrm{P}$ is true (i.e. he knows that the proposition named Pythagoras Theorem is true). Similarly, in Zemach's opinion the only possibility of interpretation of formula $K \neg K p$ is de re. John may know that he does not know that $p$, since he refers to proposition $\mathrm{P}$ through its name, not knowing the content. This conceptualization does not lead to a paradox. ${ }^{4}$

Kvanvig $(1995$; 2006) makes an emphasis on a different problem in the paradoxical argument. In the scope of quantifier in modal contexts it is forbidden to substitute non-rigid designators. Shortly speaking, if in conjunction $p \wedge \neg K p$ the right component has not a rigid designator, it can not be substituted in formula: $\forall p(p \rightarrow \diamond K p)$. The non-rigidness argument is also improved in: BrogaARD and SALERno 2008; KenNedy 2014; PRoIETTI 2016.

Kooi (2016) taking into account an ambiguity of reading de re/de dicto, proposes a logic in which Knowability Principle is formulated as:

$$
\varphi \rightarrow\langle\lambda q \diamond K q\rangle(\varphi)
$$

where the second occurrence of $\varphi$ is outside the scope of $\diamond$. Kooi underlines that if Knowability Principle is formulated in a natural language (,If $\varphi$, then it is possible that $\varphi$ is known.”), then in fact it states that ,If $\varphi$,

\footnotetext{
${ }^{3}$ Similar approach can be found in PIETRUSZCZAK and JARMUŻEK 2018, where in the context of categorial sentences de re interpretation of modalities also came down to single operators.

${ }^{4}$ This proposal met an objection that it included: "an equivocation in 'knowing the content' of $p$, between knowing what the content is and having knowledge of which it is the content". WiLLIAMSON 2000.
} 
then $\varphi$ has the property of being knowable". This shift also allows to block the paradoxical argument.

In general a strategy de re can be applied to possibility, knowledge or knowability operators (complex or sui generis). Moreover, the strategy can include different understanding of the distinction between de re/de dicto, e.g. either syntactic, semantic, or metaphysical (see NELSON 2019).

A fundamental difference between former approaches and what is proposed here is that we introduce a separate knowability operator, so the knowability is treated as sui generis with the de re interpretation. The former proposals based on a more complex interpretation of knowability, displaying erroneous steps in the paradoxical argument (particularly concerning nonrigidness), the faulty formulation of Knowability Principle, and consequently they assume a more complex language. Also our idea is to formalize Knowability Pronciple in an alternative way to solve Fitch Paradox, but at the same the solution seems much simpler. ${ }^{5}$

\section{LOGIC OF KNOWABILITY}

Our goal is to present logic with a modified knowability principle and the rest of required properties that KFP possesses and to prove that within this logic the paradox does not occur. Precisely, we need to show that the formula $\varphi \rightarrow K \varphi$ is not derivable from the empty set of premises in our logic. To do that, we need some kind of semantic structures and at least a soundness theorem (in fact we will show a completeness also).

A certain remark needs to be made here. As it was mentioned before, the kind of logics for which it is the easiest to find adequate semantics are normal modal logics. For that reason we will confine our investigation to normal modal logic which is in a way stronger than needed. What is more problematic, it yields some idealised notion of knowledge - the one in which an agent is logically omniscient. Logical omniscience however, is a very complex problem itself with no consensus concerning its overcoming (consult Meyer 2001, 190-195; Meyer and VAN Der HoEK 1995, 71-89; Fagin, HALPERN, MOSES, and VARDI 1995, 309-347). Since there is no universal framework which would be logically efficient for our purposes, we decide to stick to normal modal epistemic logic which unfortunately presupposes logical

\footnotetext{
${ }^{5}$ Our standpoint is very to close to understanding of knowability as potenitial knowledge, see FUHRMANN 2014.
} 
omniscience. What is more, some may state that solving logical omniscience is the key to overcoming Fitch paradox: if we reject $K$ distribution over conjunction and hence reject omniscience, we cannot perform the derivation. We claim that the paradox can be avoided with preservation of logically idealised notion of knowledge. What is needed, however, is the notion of possible knowledge, which is something different, though strictly related to the idealised knowledge.

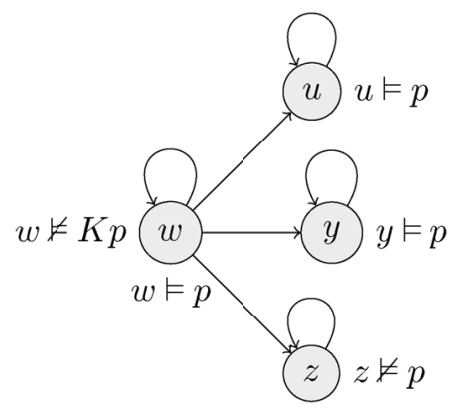

Figure 1: Model $\mathfrak{M}$

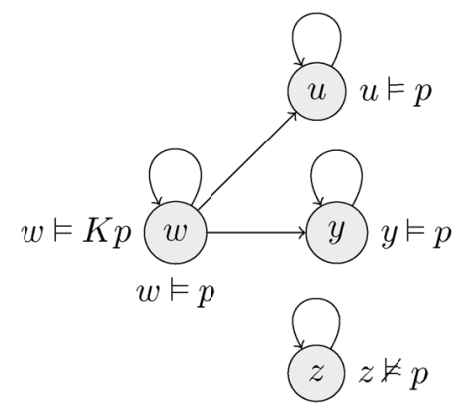

Figure 2: Model $\mathfrak{M}^{\prime}$

Here we state some general intuitions behind our proposal. Firstly, possible knowledge, the knowledge which is obtainable somewhere, sometime by someone contains actual knowledge, the one which is already in possession. The motivation behind this constatation is the following: since the knowledge has been obtained (by someone), it is obtainable (by someone else). Formally, the formula $K p \rightarrow K^{\diamond} p$ expresses this statement, if we use $K^{\diamond}$ to denote possible knowledge/knowability operator. We want this formula to be a thesis of our future system.

Secondly, we need to make some comments about possible world framework in epistemic logic. An agent's accessability relation in a model represents his doubt rather than his knowledge. The more epistemic alternatives, the bigger the chance that some sentence will be falsified in some world. To see that, let us consider two single agent models with epistemic ac:cessibility relations $R$ and $R^{\prime}$ :

$$
\begin{aligned}
& \mathfrak{M}=\left\langle W^{\prime}, R^{\prime}, V^{\prime}\right\rangle, \text { where } W^{\prime}=\{w, u, y, z\}, \\
& R^{\prime}=\{\langle w, u\rangle,\langle w, y\rangle,\langle w, z\rangle,\langle w, w\rangle,\langle u, u\rangle,\langle y, y\rangle,\langle z, z\rangle\}, \\
& V^{\prime}(p)=\{w, u, y\} \text { and for any }, \varphi \in \operatorname{Var} \text { if } \varphi \neq p, \text { than } V^{\prime}(\varphi)=\varnothing \text { (Figure 1), }
\end{aligned}
$$




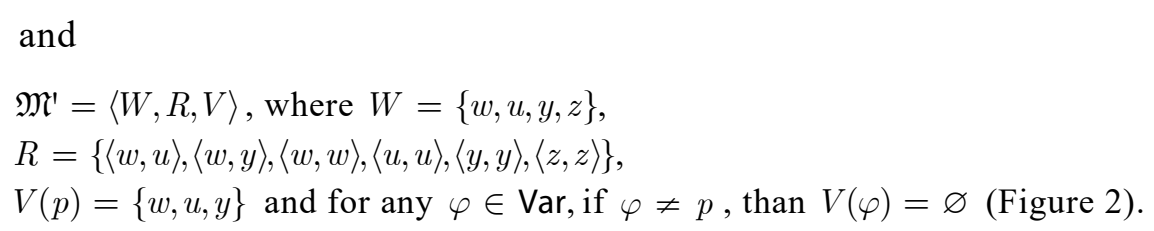

We can see that in model $\mathfrak{M}^{\prime}$ agent knows $p$, while in $\mathfrak{M}$, he does not know $p$ any more. In this sense, we can say that the possible world framework models doubt rather than knowledge. Hence, an extension of a number of epistemic alternatives resulted in the decrease of agents knowledge. The less alternatives - the bigger the knowledge.

How does it affect our previous remarks on relation between knowledge and knowability? If we were to include both modalities in possible-world frame, we would like to have two separate binary relations for different types of knowledge. The epistemic relation for $K^{\diamond}$ - lets call it $R^{\diamond}$ - should turn out to be a special subrelation of $K$ 's $-R$. This shall be the case if we want possible knowledge to contain those propositions that could be known, only if the falsifying worlds were to be removed from a model. This way, the possible knowledge would still contain the actual knowledge. For example, in our model $\mathfrak{M}$ we have $\mathfrak{M}, w \vDash \neg K p$, but we could have $\mathfrak{M}, w \vDash K^{\diamond} p$, if we had removed the pair $\langle w, z\rangle$ from $R$ - thus obtaining $R^{\diamond}$.

\section{AXIOMATIZATION}

We want to obtain normal modal logic for antirealism eg. the one in which knowledge factivity and knowability principle holds. We can call it a logic of knowability, since - to express possible knowledge - we introduce knowability operator $K^{\diamond}$ and interpret it as a certain kind of knowledge. However, we use abbreviation ARL from word anti-realism, because it incorporates Knowalibity Principle as its thesis.

Our language $\mathcal{L}_{\mathrm{ARL}}$ consists of the set of propositional variables $\mathrm{Var}=$ $\left\{p_{0}, p_{1}, \ldots\right\}$, set of classical connectives $\{\neg, \wedge, \vee, \rightarrow, \leftrightarrow\}$ and set of unary modalities $\left\{K, K^{\diamond}\right\}$. The set of formulas over $\mathcal{L}_{\mathrm{ARL}}$ is build in the standard inductive way and will be referred to as $\operatorname{FOR}\left(\mathcal{L}_{\mathrm{ARL}}\right)$.

We assume the following axioms and rules: 
(Taut) All tautologies of CPL expressed in $\operatorname{FOR}\left(\mathcal{L}_{\mathrm{ARL}}\right)$

$$
\begin{aligned}
& (K) \quad K(\varphi \rightarrow \psi) \rightarrow(K \varphi \rightarrow K \psi) \\
& \left(K^{\diamond}\right) K^{\diamond}(\varphi \rightarrow \psi) \rightarrow\left(K^{\diamond} \varphi \rightarrow K^{\diamond} \psi\right) \\
& (K F a c) K \varphi \rightarrow \varphi \\
& \left(K^{\diamond} p\right) \varphi \rightarrow K^{\diamond} \varphi \\
& \quad(M P) \\
& \quad \frac{\varphi \rightarrow \psi, \varphi}{\psi}
\end{aligned}
$$

Definition 4.1 (ARL-proof). The ARL-proof of formula $\varphi$ is a sequence $\psi_{1}, \ldots, \psi_{n}$, where $\psi_{n}=\varphi$, and for each $i \in\{1, \ldots, n\}, \psi_{i}$ fulfils at least one of the following:

- $\psi_{i}$ is an axiom,

- there are $j, k<i$, such that $\psi_{j}=\psi_{k} \rightarrow \psi_{i}$,

- there is $j<i$, such that $\psi_{i}=K \psi_{j}$,

If there is an ARL -proof of the formula $\varphi$, we will denote it by $\vdash_{\text {ARL }} \varphi$. By ARL logic we understand $\left\{\varphi: \vdash_{\text {ARL }} \varphi\right\}$. We will write simply ARL to denote the ARL logic.

Definition 4.2 Let $\Gamma$ be a set of formulas, $\varphi$ a formula. We say that $\varphi$ is deducible from $\Gamma$ in ARL (symbolically: $\Gamma \nvdash_{A R L} \varphi$ ) iff there are $\psi_{1}, \ldots, \psi_{n} \in \Gamma$ such that $\vdash_{A R L} \psi_{1} \wedge \ldots \wedge \psi_{n} \rightarrow \varphi$.

Actually, we can prove that the rule for $K^{\diamond}$ which is analogous to $($ Knec $)$ is derivable in our logic.

Fact 4.3 The rule $\left(K^{\diamond} n e c\right) \frac{\varphi}{K^{\diamond} \varphi}$ is deducible in ARL.

Proof. By $\left(K^{\diamond} P\right)$ and $(M P)$.

\section{SEMANTICS}

In order to obtain some interpretation for our logic we introduce a notion of possible world semantics model. Since we have two kinds of unary modalities in our logic we make use of two binary relations $R$ and $R^{\diamond}$ for standard epistemic modality $K$ and for possible knowledge modality $K^{\diamond}$ respectively.

Our model $\mathfrak{M}$ is a quadruple: $\mathfrak{M}=\left\langle W, R, R^{\diamond}, V\right\rangle$, where $W \neq \varnothing$, $R, R^{\diamond} \subseteq W \times W$ and $V: \operatorname{Var} \rightarrow P(W)$. The underlying frame is a triple: 
$\mathfrak{F}=\left\langle W, R, R^{\diamond}\right\rangle$.

We assume the following truth-conditions in a model for the formulas in respect of their complexity:

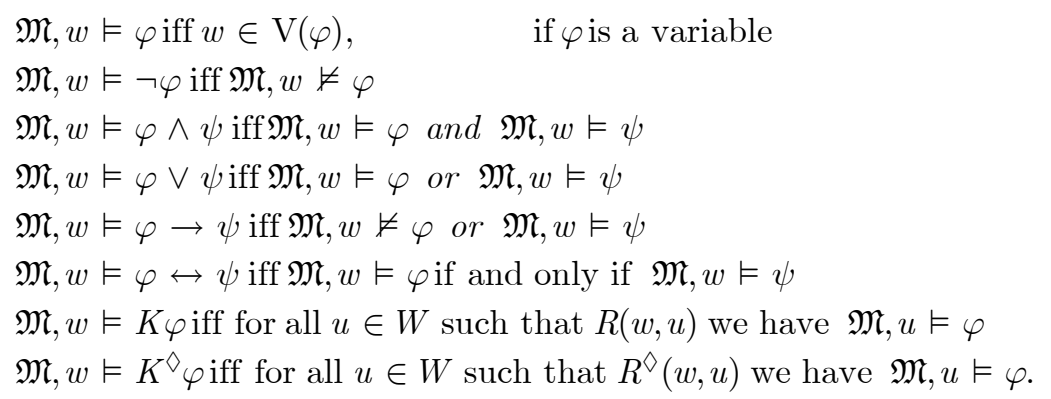

We say that a formula is true in a model if it is true in all worlds from this model. A formula is valid on a frame if it is true in all models built on that frame. Finally, we say that a formula is valid in a class of frames if it is true in every frame from that class. It is quite obvious that for modal logics with two unary operators $K$ and $K^{\diamond}$ we need to consider classes of frames with two binary relations. What is more, (KFac) determines the class of frames with reflexive $R$. What we are interested in, is the condition that is imposed on the frames by the formula $\left(K^{\diamond} P\right)$, if any.

Theorem 5.1 $\left(K^{\diamond} P\right)$ determines the frames with the following first-order property: $\forall_{x, y \in W}\left(R^{\diamond}(x, y) \Rightarrow x=y\right)$.

Proof. Let $\mathfrak{F}=\left\langle W, R, R^{\diamond}\right\rangle$.

$(\Rightarrow)$ We take such $x, y \in W$ that $R^{\diamond}(x, y)$ and assume that $x \neq y$. Let $V$ be such that $V(p)=\{x\}$. Since $\langle\mathfrak{F}, V\rangle, y \not \models p$, we get $\langle\mathfrak{F}, V\rangle, x \not \models p \rightarrow K^{\diamond} p$. As a consequence $\mathfrak{F} \not \models \varphi \rightarrow K^{\diamond} \varphi$.

$(\Leftarrow)$ Assume $\mathfrak{F} \not \models \varphi \rightarrow K^{\diamond} \varphi$. Hence, there is $\mathfrak{M}=\langle\mathfrak{F}, V\rangle$ such that $\mathfrak{M}, w \vDash \varphi$ and $\mathfrak{M}, w \not \models K^{\diamond} \varphi$. So there is $u \in W$ such that $R^{\diamond}(w, u)$ and $\mathfrak{M}, u \not \models p$. Obviously $w \neq u$.

The class of frames $\mathfrak{F}=\left\langle W, R, R^{\diamond}\right\rangle$ where $R$ is reflexive and $R^{\diamond}$ is as above will be called $\mathrm{F}_{\mathrm{ARL}}$.

\section{ADEQUACY}

Now we are going to prove adequacy of our Hilbert style system with the class of frames $F_{A R L}$. We will start from soundness theorem. 
Theorem 6.1 (Soundness) $\vdash_{A R L} \varphi$ implies $F_{A R L} \vDash \varphi$.

Proof. By $5.1\left(K^{\diamond} P\right)$ is valid on $\mathrm{F}_{\mathrm{ARL}}$. The rules preserves validity. The rest of the axioms are obviously valid on $\mathrm{F}_{\mathrm{ARL}}$.

Having proven the soundness we can move on to the completeness. To prove the theorem, we will start from the definitions of ARL-consistent set and $A R L$-maximal consistent set of formulas.

Definition 6.2 (Maximal consistent set) Let $\Gamma$ be a set of formulas.

- $\Gamma$ is ARL-consistent iff $\Gamma \nvdash_{A R L} \varphi \wedge \neg \varphi$; otherwise, it is inconsistent;

- $\Gamma$ is ARL-maximal consistent set (ARL-mes) iff it is ARL-consistent and its any proper extension is inconsistent.

Now we are ready to show the well-known Lindenbaum's lemma.

Lemma 6.3 (Lindenbaum's Lemma)

Any ARL-consistent set of formulas $\Sigma$ can be extended to ARL-maximal consistent set (ARL-mcs).

Proof. Let $\varphi_{0}, \varphi_{1}, \ldots$ be a sequence of all formulas. We define the sequence of set of formulas in the following way:

$$
\begin{aligned}
& \Sigma_{0}=\Sigma \\
& \Sigma_{n+1}=\left\{\begin{array}{cc}
\Sigma_{n} \cup\left\{\varphi_{n}\right\}, & \text { if } \Sigma_{n} \cup\left\{\varphi_{n}\right\} \nvdash_{\text {ARL }} \phi \wedge \neg \phi ; \\
\Sigma_{n} \cup\left\{\neg \varphi_{n}\right\}, & \text { otherwise. }
\end{array}\right. \\
& \Sigma^{+}=\bigcup_{n \geq 0}^{\infty} \Sigma_{n} .
\end{aligned}
$$

Now we need to proof that $\Sigma^{+}$is indeed an extension of $\Sigma$ and an ARL - mcs. Obviously $\Sigma \subseteq \Sigma^{+}$, so $\Sigma^{+}$is an extension of $\Sigma$. For the second part we need to show that 1) $\Sigma^{+} \nvdash_{\text {ARL }} \phi \wedge \neg \phi$ and 2) any proper extension of $\Sigma^{+}$is ARL-inconsistent. For 1) assume $\Sigma^{+} \vdash_{\text {ARL }} \phi \wedge \neg \varphi$. Than, there are $\sigma_{1}, \ldots, \sigma_{n} \in \Sigma^{+}$such that $\vdash_{\text {ARL }} \sigma_{1} \wedge \ldots \wedge \sigma_{n} \rightarrow \varphi \wedge \neg \varphi$. It means that there is $\Sigma_{k}$ such that for all $i \in\{1, \ldots, n\}, \sigma_{i} \in \Sigma_{k}$. Hence $\Sigma_{k}$ is inconsistent, but this is impossible from the above sequence construction. Contradiction, hence $\Sigma^{+}$ is ARL-consistent. For 2) assume there is a proper extension $\Gamma$ of $\Sigma^{+}$and $\Gamma \nvdash_{A R L} \phi \wedge \neg \phi$. There must be some formula $\psi$ such that $\psi \in \Gamma$ and $\psi \notin \Sigma^{+} . \psi$ must appear somewhere in the sequence, so there is $n \in \mathbb{N}$ such that $\psi=\varphi_{n}$. By the construction: $\psi \in \Sigma^{+}$or $\neg \psi \in \Sigma^{+}$. If $\psi \in \Sigma^{+}$, than $\Gamma$ is 
not the proper extension of $\Sigma^{+}$. If $\neg \psi \in \Sigma^{+}$, than $\Gamma$ is ARL-inconsistent. Either way it contradicts the assumptions.

We can state some facts concerning maximally consistent sets that will turn out useful in later investigations:

Fact 6.4 The following hold for any $A R L-m c s \Sigma^{+}$:

- $\varphi, \psi \in \Sigma^{+}$iff $\varphi \wedge \psi \in \Sigma^{+}$

- if $\varphi \in \Sigma^{+}$and $\vdash_{A R L} \varphi \rightarrow \psi$, than $\psi \in \Sigma^{+}$.

Both facts can be easily proven from the construnction of $A R L-m c s$.

Here, we are ready to define the canonical model for our logic, which is a crucial structure for proving completeness.

Definition 6.5 (Canonical model) Let $W_{\Lambda}$ be the set of all ARL-mcs.

Let for all $x, y \in W_{\Lambda}$ :

- $R_{\Lambda}(x, y)$ iff $K \varphi \in x$ implies $\varphi \in y$, for any $\varphi$

- $R_{\Lambda}^{\diamond}(x, y)$ iff $K^{\diamond} \varphi \in x$ implies $\varphi \in y$, for any $\varphi$.

The canonical model for $\vdash_{A R L}$ is $\mathfrak{M}_{\Lambda}=\left\langle W_{\Lambda}, R_{\Lambda}, R_{\Lambda}^{\diamond}, V_{\Lambda}\right\rangle$, where

- $R_{\Lambda} \subseteq W_{\Lambda} \times W_{\Lambda}$ is the $K$-accessibility relation,

- $R_{\Lambda}^{\diamond} \subseteq W_{\Lambda} \times W_{\Lambda}$ is the $K^{\diamond}$-accessibility relation,

- $V_{\Lambda}: \operatorname{Var} \rightarrow \mathcal{P}\left(W_{\Lambda}\right)$ is a valuation such that $x \in V_{\Lambda}(\varphi)$ iff $\varphi \in x$, for any $\varphi \in$ Var.

Lemma 6.6 (Existence lemma) For all $x \in W_{\Lambda}$ :

(K) If $K \varphi \notin x$, than there is $y \in W_{\Lambda}$ such that $R_{\Lambda}(x, y)$ and $\varphi \notin y$,

$\left(K^{\diamond}\right)$ If $K^{\diamond} \varphi \notin x$, than there is $y \in W_{\Lambda}$ such that $R_{\Lambda}^{\diamond}(x, y)$ and $\varphi \notin y$.

Proof. We prove the lemma only for $(K)$ since the case for $\left(K^{\diamond}\right)$ is analogous.

Let $x \in W_{\Lambda}, \varphi$ be arbitrary. Assume $K \varphi \notin x$. First, we show that $\{\neg \varphi\} \cup$ $\{\psi: K \psi \in x\} \nvdash_{A R L} \phi \wedge \neg \phi$. Assume otherwise:

$\{\neg \varphi\} \cup\{\psi: K \psi \in x\} \vdash_{A R L} \phi \wedge \neg \phi$. From CPL we get $\vdash_{\text {ARL }} \psi_{1} \wedge \ldots \wedge \psi_{n} \rightarrow \varphi$, where for each $i \in\{1, \ldots, n\}, \psi_{i} \in\{\psi: K \psi \in x\}$. Since $A R L$ is a normal extension of modal logic $K$ we get $\vdash_{\text {ARL }} K \psi_{1} \wedge \ldots \wedge K \psi_{n} \rightarrow K \varphi$. From 6.4 we get $K \varphi \in x$. A contradiction, hence $\{\neg \varphi\} \cup\{\psi: K \psi \in x\}$ is $A R L-$ consistent . The set we denote by $\Sigma$.

By (6.3) $\Sigma$ can be extended to $A R L-m c s$. Such extension will be called $\Sigma^{+}$. By definition (6.4) $\Sigma^{+} \in W_{\Lambda}$ and by (6.5) $R_{\Lambda}\left(x, \Sigma^{+}\right)$. Moreover, 
$\neg \varphi \in \Sigma^{+}$, so by $(6.3) \varphi \notin \Sigma^{+}$. Hence, $\Sigma^{+}$is our $y$.

Lemma 6.7 (Truth lemma) $\varphi \in x$ iff $\mathfrak{M}_{\Lambda}, x \vDash \varphi$, for all $x \in W_{\Lambda}$.

Proof. By induction on the complexity of $\varphi$. We will omit the cases for boolean connectives and focus only on case with $K$, since the proof for $K^{\diamond}$ is analogous. Base case from (6.5).

Inductive step: Let $\varphi:=K \psi$. First assume $K \psi \in x$. Let $y \in W_{\Lambda}$ be arbitrary. Assume $R_{\Lambda}(x, y)$. By (6.5) $\psi \in y . \mathfrak{M}_{\Lambda}, y \vDash \psi$ from inductive hypothesis. Hence $\mathfrak{M}_{\Lambda}, x \vDash K \psi$. For the other direction assume $K \psi \notin x$. By (6.6) there is $y \in W_{\Lambda}$ such that $R_{\Lambda}(x, y)$ and $\psi \notin y$. Let $a$ be such $y$. $\mathfrak{M}_{\Lambda}, a \not \models \psi$ from hypothesis. Hence $\mathfrak{M}_{\Lambda}, x \not \models K \psi$.

Now we are in a position to prove the completeness result. First we state that $\nvdash_{A R L} \varphi$ implies $F_{A R L} \not \models \varphi$ - the succedent means that there are such $\mathfrak{F}=\left\langle W, R, R^{\diamond}\right\rangle \in \mathrm{F}_{\text {ARL }}, \mathfrak{M}=\langle\mathfrak{F}, V\rangle, w \in W$ that $\mathfrak{M}, w \not \models \varphi$. To show that we are going to prove two lemmas.

Lemma 6.8 $\nvdash_{A R L} \varphi$ implies that $\mathfrak{M}_{\Lambda} \not \models \varphi$.

Proof. Assume $\nvdash_{A R L} \varphi$. By CPL $\{\neg \varphi\} \nvdash_{A R L} \phi \wedge \neg \phi$, hence by (6.3) we can extend $\{\neg \varphi\}$ to $A R L-m c s$. Let $x$ be such a $A R L-m c s$. By (6.5) $x \in W_{\Lambda}$ and by (6.7) $\mathfrak{M}_{\Lambda}, x \vDash \neg \varphi$, hence $\mathfrak{M}_{\Lambda}, x \not \models \varphi$.

Lemma 6.9 $\mathfrak{F}_{\Lambda}=\left\langle W_{\Lambda}, R_{\Lambda}, R_{\Lambda}^{\diamond}\right\rangle \in F_{A R L}$.

Proof. We have to show that $R_{\Lambda}$ is reflexive and $R_{\Lambda}^{\diamond}$ meets the condition: $\forall_{x, y \in W}\left(R^{\diamond}(x, y) \Rightarrow x=y\right)$. Let $x \in W_{\Lambda}$ be arbitrary.

Let $\varphi$ be any formula such that $\varphi:=K \psi$ and $\varphi \in x . K \psi \rightarrow \psi \in x$, hence $\psi \in x$ by (6.4) which by definition (6.5) means $R_{\Lambda}(x, x) . R_{\Lambda}$ is reflexive.

For the second part assume $y$ is such that $R_{\Lambda}^{\diamond}(x, y)$ and $x \neq y$, which means that there is $\varphi$ such that $\varphi \in x$ and $\varphi \notin y$. Let $\psi$ be such $\varphi$. $\psi \rightarrow K^{\diamond} \psi \in x$ so $K^{\diamond} \psi \in x$ by (6.4) $\psi \in y$ from definition (6.5). Contradiction, hence $x=y$ as required.

Finally, we can prove the completeness theorem.

Theorem 6.10 (Completeness) $F_{\text {ARL }} \vDash \varphi$ implies $\vdash_{\text {ARL }} \varphi$.

Proof. By (6.8) and (6.9)we get $\nvdash_{\text {ARL }} \varphi$ implies $\mathrm{F}_{\mathrm{ARL}} \not \models \varphi$, hence if $\mathrm{F}_{\mathrm{ARL}} \vDash \varphi$, than $\vdash_{\text {ARL }} \varphi$. 


\section{COUNTERMODELS FOR FITCH PARADOX}

Now we are going to refute the Fitch Paradox in $\vdash_{\text {ARL }}$. To show that the paradoxical reasoning can not be reproduced in our logic, we will make use of completeness theorem and prove that $\nvdash_{\text {ARL }} p \rightarrow K p$.

Theorem 7.1 $F_{\text {ARL }} \not \models \varphi \rightarrow K \varphi$

Proof. It is sufficient to find $\mathfrak{F}=\left\langle W, R, R^{\diamond}\right\rangle \in \mathrm{F}_{\text {ARL, }}, \mathfrak{M}=\langle\mathfrak{F}, V\rangle$ and $w \in W$ such that $\mathfrak{M}, w \not \models p \rightarrow K p$. In order to do that, we build countermodel $\mathfrak{M}_{*}=\left\langle W_{*}, R_{*}, R_{*}^{\diamond}, V_{*}\right\rangle$, where $W_{*}=\{u, w\}, \quad R_{*}=\{\langle u, u\rangle,\langle w, w\rangle,\langle u, w\rangle\}, \quad R_{*}^{\diamond}=\{\langle u, u\rangle$, $\langle w, w\rangle\}$ and $V_{*}$ is such that $V_{*}(p)=\{u\}$ and for each $\varphi \in \operatorname{Var}$, if $\varphi \neq p$, than $V_{*}(\varphi)=W_{*}$. Our model has the required properties and falsifies the formula $p \rightarrow K p$ at the world $u$. By $V_{*}$ we have $\mathfrak{M}_{*}, u \vDash p$. Since $\mathfrak{M}_{*}, w \not \models p$ and $R_{*}(u, w)$ we get $\mathfrak{M}_{*}, u \not \models K p$.

By (7.1)and the soundness we get $\nvdash_{\text {ARL }} p \rightarrow K p$. It would be interesting to come back to the original Fitch's proof that we presented in the beginning and see where is the step in which the derivation would fail in $\vdash_{A R L}$. Obviously, it is for example step (6), because we do not have the necessity operator in our logic.

Now let us get back to our intuitions. Obviously:

$$
\vdash_{\text {ARL }} K p \rightarrow K^{\diamond} p
$$

by $($ KFac $),\left(K^{\diamond} P\right)$, and CPL. By completeness:

$$
\mathrm{F}_{\mathrm{ARL}} \vDash K p \rightarrow K^{\diamond} p
$$

hence, in all our frames $R^{\diamond} \subseteq R$.

What may seem a kind of counter-intuitive is the knowability of sentences that can not be true. For example, the formula $K^{\diamond}(p \wedge \neg p)$ is satisfiable in $F_{A R L}$. In order to show this, we consider any model based on the frame from $F_{A R L}$ with empty $R^{\diamond}$.

We see few possibilities to overcome this difficulty. One of them is to enhance $\left(K^{\diamond} P\right)$ to:

$$
\left(K^{\diamond} P^{\prime}\right) \varphi \leftrightarrow K^{\diamond} \varphi
$$

In terms of semantics we need the class of frames $F_{A R L}$, where $R^{\diamond}$ is restricted by the condition: $\forall_{x, y \in W}\left(R^{\diamond}(x, y) \Leftrightarrow x=y\right)$, so $R^{\diamond}$ should be the least reflexive relation. This subtlety is only a little part of the story we would like to tell in the future, when we examine some of other possible extensions of $\vdash_{A R L}$. 


\section{TABLEAUX APPROACH TO $\vdash_{\text {ARL }}$}

Now, we shall outline the tableaux approach to our logic. We will be governed here by a strategy adopted in paper by Jarmużek (2013) which introduced a formalized tableaux theory for some modal logics. Let us, however, disregard the formal concepts in favour of stressing the crucial points which determine the completeness of the tableaux approach related to the semantically designated consequence relation.

For current investigations, we shall need a new language. A language of tableaux proofs. As we know, a tableaux proof for a logic determined by possible worlds semantics is usually carried out in a set of formulas with labels that are natural numbers. We would like to call them indexes. Let $\mathbb{N}$ be the set of natural numbers. A set of tableaux expressions $E x$ is a union of the following sets:

- $\{i r j: i, j \in \mathbb{N}\}$

- $\left\{i r^{\diamond} j: i, j \in \mathbb{N}\right\}$

- $\operatorname{FOR}\left(\mathcal{L}_{\mathrm{ARL}}\right) \times \mathbb{N}$.

In all cases it is possible we will omit the brackets: $\langle$,$\rangle , so for example$ instead of $\langle\varphi, i\rangle$, we will just write $\varphi, i$ etc. Let us now explain what the particular expressions are intended to encode. Expressions of the form irj naturally encode in the tableaux language an accessibility relation $R$ between worlds denoted by $i$ and $j$. Expressions of the form $i r^{\diamond} j$ encode in the tableaux language an accessibility relation $R^{\diamond}$ between worlds denoted by $i$ and $j$. Surely, expressions $\varphi, i$ traditionally encode that a formula $\varphi$ is true at a world denoted by $i$.

Now, all tableaux proofs are carried out in language Ex. A tableaux inconsistent set of expressions (that closes a given branch) is the one comprising a pair:

- $\varphi, i$ and $\neg \varphi, i$,

for some $\varphi \in \operatorname{FOR}\left(\mathcal{L}_{\mathrm{ARL}}\right)$ and $i, j \in \mathbb{N}$. We say that a set of tableaux expressions is tableaux consistent iff it is not a tableaux inconsistent set.

We propose a set of tableaux rules for our logic. For the formulas with main classical connectives: $\neg, \wedge, \vee, \rightarrow, \leftrightarrow$, we shall assume the standard tableaux rules. We do not need to list or elaborate them as they have been thoroughly examined in many papers (see GORÉ 1999; JARMUŻEK 2013).

The modality of knowledge $K$ behaves like a modality in alethic modal $\operatorname{logic}$ T. So for $K$ we assume the following standard tableaux rules: 


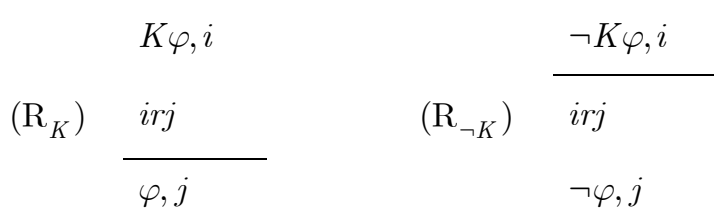

where rule $\left(\mathrm{R}_{\neg K}\right)$ is obviously limited by clause that index $j$ is new on the branch. Moreover, since accessibility relation $R$ in a model is assumed to be reflexive, we add also a rule:

$$
\left(\mathrm{R}_{\text {ref }}\right) \frac{}{i r i}
$$

where $i$ previously appeared on the branch.

The modality of possible knowledge $K^{\diamond}$ behaves at the basic level like a modality in alethic modal logic $\mathrm{K}$, but with some later modification. So for $K^{\diamond}$ we assume the following two standard tableaux rules:

$$
\begin{array}{rrr}
\left.K_{K^{\diamond}}\right) & & \frac{\neg K^{\diamond} \varphi, i}{i r^{\diamond} j} \\
\hline \varphi, j & \left(\mathrm{R}_{\neg K^{\diamond}}\right) & i r^{\diamond} j \\
& & \neg \varphi, j
\end{array}
$$

where rule $\left(\mathrm{R}_{\neg K^{\diamond}}\right)$ is obviously limited by clause that index $j$ is new on the branch.

Additionally, since $R^{\diamond}$ is supposed to satisfy in a model condition: ${ }_{x, y \in W}\left(R^{\diamond}(x, y) \Rightarrow x=y\right)$, so we add a new rule:

$$
\begin{aligned}
& i r^{\diamond} j \\
& \left(\mathrm{R}_{\mathrm{ARL}}\right) \frac{\varphi, j}{\varphi, i}
\end{aligned}
$$

The rule $\left(\mathrm{R}_{A R L}\right)$ is the only one among the listed ones that must be extensively inspected in the context of soundness and completeness of the proposed here tableaux approach to $\vdash_{\text {ARL }}$.

The set of all mentioned rules will be denoted as $\mathbf{R}$.

For the simplification, let us call the expressions in a tableaux rule numerator input, while those in denominator output. Some rules, e.g. among those for the classical connectives may have more than one output, since they generate more than only one branch. 
Let us now introduce two concepts which are important for the tableaux issues.

Definition 8.1 (Set of indexes). Let $X \subseteq \mathrm{Ex}$. By function Ind $: X \rightarrow P(\mathbb{N})$ we mean a mapping satisfying conditions:

- if $X=\{$ irj $\}$, then $\operatorname{Ind}(X)=\{i, j\}$, for all $i, j \in \mathbb{N}$

- if $X=\left\{i r^{\diamond} j\right\}$, then $\operatorname{Ind}(X)=\{i, j\}$, for all $i, j \in \mathbb{N}$

- if $X=\{\langle\varphi, i\rangle\}$, then $\operatorname{Ind}(X)=\{i\}$, for all $i \in \mathbb{N}$ and $\varphi \in \operatorname{FOR}(\mathcal{L})$

- $\operatorname{lnd}(X)=\bigcup\{\operatorname{lnd}(y): y \in X\}$.

Any function Ind collects indexes contained in expressions from a given subset of Ex.

Now, we can extend in a certain sense the concept of truth in a model from the formulas to all expressions from Ex.

Definition 8.2 (Model suitable to a set of expressions). Let $\mathfrak{M}=\left\langle W, R, R^{\diamond}, V\right\rangle$ be a model and $X \subseteq E x$. Model $\mathfrak{M}$ is suitable to $X$ iff there exists a function $f$ from the set of indexes contained in expressions from $X$ to $W$, i.e. $f: \operatorname{Ind}(X) \rightarrow W$, such that, for any $\varphi \in \operatorname{FOR}(\mathcal{L})$ and $i, j \in \mathbb{N}$ :

- if irj $\in X$, then $R(f(i), f(j))$

- if $i r^{\diamond} j \in X$, then $R^{\diamond}(f(i), f(j))$

- if $\langle\varphi, i\rangle \in X$, then $\mathfrak{M}, f(i) \vDash \varphi$.

Making use of the provided concept of a suitable model $\mathfrak{M}$ and conducting an inspection of the provided tableaux rules, we are able to demonstrate that if a model is suitable for set of expressions $X \subseteq \mathrm{Ex}$, then an application of a selected tableaux rule extends set $X$ with a new expression or expressions for which model $\mathfrak{M}$ is still suitable.

Let us now phrase a proposition.

Lemma 8.3 (Rules sound to model). Let:

- $X \subseteq E x$

- $\mathfrak{M}=\left\langle W, R, R^{\diamond}, V\right\rangle$ be a model suitable to $X$.

If any tableaux rule from $R$ has been applied to set $X$, then $\mathfrak{M}$ is suitable to union of $X$ and at least one output obtained through application of this rule.

Proof. Assume all hypothesis. Let $f: \operatorname{lnd}(X) \rightarrow W$ be a function as in definition (8.2). For cases of applications of most rules from set $\mathbf{R}$ the proof is standard (see GORÉ 1999; JARMUŻEK 2013). 
A new case is the case of rule $\left(\mathrm{R}_{\mathrm{ARL}}\right)$. If it has been applied to $X$, then $i r^{\diamond} j,\langle\varphi, j\rangle \in X$, and by assumption about $f, \quad R^{\diamond}(f(i), f(j))$ and $\mathfrak{M}, f(j) \vDash \varphi$. However, since $R^{\diamond}(f(i), f(j)$ ), so $f(i)=f(j)$ (by condition for the frames from $\left.\mathrm{F}_{\mathrm{ARL}}\right)$, and therefore $\mathfrak{M}, f(i) \vDash \varphi$, which means that $\mathfrak{M}$ is suitable to $X \cup\{\langle\varphi, i\rangle\}$, by definition (8.3).

The proof of completeness of our tableaux methods in relation to the presented semantics still requires a converse proposition in a sense. Let us introduce a concept of a model produced by a set of expressions.

Definition 8.4 (Model generated by branch). Let $X \subseteq E x$. Model $\left\langle W, R, R^{\diamond}, V\right\rangle$ is generated by $X$ iff

- $W=\{i: i \in \operatorname{Ind}(X)\} \backslash\left\{j: \exists_{i \in \mathbb{N}} i r^{\diamond} j \in X\right\}$

- $R(i, j)$ iff $i r j \in X$, for all $i, j \in W$

- $R^{\diamond}(i, k)$ iff $\exists_{j}\left[i r^{\diamond} j \in X\right]$ and $i=k$, for all $i, k \in W$

- for every $\varphi \in \operatorname{Var}, i \in W: i \in V(\varphi)$ iff $\langle\varphi, i\rangle \in X$.

Now, if we take a set of expressions $X \subseteq$ Ex such that:

- it is closed under all rules from $\mathbf{R}$ - for all expressions from $X$ to which one of the rules is applicable, there exists exactly one output in $X$

- $X$ is a tableaux consistent set of expressions,

then there is a model $\mathfrak{M}$ generated by set $X$. Therefore, we have one more proposition.

We have a fact that generating from closed and consistent sets of expressions gives models that are underlined by frames from $\mathrm{F}_{\mathrm{ARL}}$.

Fact 8.5 Let $X \subseteq$ Ex be a closed under tableaux rules $\boldsymbol{R}$ and tableaux consistent set of expressions. Let $\mathfrak{M}=\left\langle W, R, R^{\diamond}, V\right\rangle$ be the model generated by $X$. Then $\left\langle W, R, R^{\diamond}\right\rangle \in F_{A R L}$.

Proof. To obtain the thesis we must show that:

1. $\forall_{x \in W} R(x, x)$

2. $\forall_{x, y \in W}\left(R^{\diamond}(x, y) \Rightarrow x=y\right)$.

For 1. we observe that since $X$ is closed under tableaux rules $\mathbf{R}$ and is a tableaux consistent set, so by rule $\left(\mathrm{R}_{\text {ref }}\right)$, for all $i \in \operatorname{lnd}(X)$, we have iri $\in X$. Hence, by definition of $R$ and $W$ in (8.4), $\forall_{x \in W} R(x, x)$.

For 2. we assume that $R^{\diamond}(a, b)$, for $a, b \in W$. Then by definition of $R^{\diamond}$ in (8.4), $a=b$. 
Lemma 8.6 (Model sound to rules). Let $X \subseteq$ Ex be a tableaux consistent set. If $X$ is closed under tableaux rules $\boldsymbol{R}$, then there is model $\mathfrak{M}=\left\langle W, R, R^{\diamond}, V\right\rangle$ such that for any $\langle\varphi, i\rangle \in X \cap\left(\operatorname{FOR}\left(\mathcal{L}_{\mathrm{ARL}}\right) \times W\right), \mathfrak{M}, i \vDash \varphi$.

Proof. We conduct a proof by induction on the complexity of expressions contained in $X$. We consider only the expressions that include indexes from $W$.

The initial step is for letters and negated letters. Let $\langle\varphi, i\rangle \in X \cap(\operatorname{Var} \times W)$, for some $i \in \mathbb{N}$. Then by definition of $V$ in (8.4), and so $\mathfrak{M}, i \vDash \varphi$.

Let $\langle\neg \varphi, i\rangle \in X \cap\left(\operatorname{FOR}\left(\mathcal{L}_{\mathrm{ARL}}\right) \times W\right)$, for some $i \in \mathbb{N}$, where $\varphi \in \operatorname{Var}$. Since $X$ is tableau consistent, so $\langle\varphi, i\rangle \notin X$. Then by definition of $V$ in (8.4), $i \notin V(\varphi)$, and so $\mathfrak{M}, i \not \models \varphi$. In consequence $\mathfrak{M}, i \vDash \neg \varphi$.

The inductive step. We have to assume that for any expression $\langle\varphi, i\rangle \in X \cap\left(\operatorname{FOR}\left(\mathcal{L}_{\mathrm{ARL}}\right) \times W\right)$, where $\varphi$ is of the complexity $n$, for some $n \in \mathbb{N}, \mathfrak{M}, i \vDash \varphi$. We will consider a formula $\psi$ of the complexity $n+1$.

Again the cases for classical connectives: $\neg, \wedge, \vee, \rightarrow, \leftrightarrow$ are obvious and thoroughly examined. We concentrate on the modal as well as on new cases, so we also check some cases for negation: $\neg K$ and $\neg K^{\diamond}$.

Let $\psi:=\langle K \phi, i\rangle \in X \cap\left(\operatorname{FOR}\left(\mathcal{L}_{\mathrm{ARL}}\right) \times W\right)$, or some $i \in \mathbb{N}$. Since $X$ is closed under $\left(\mathrm{R}_{\text {ref }}\right)$, so there is at least one $j \in \mathbb{N}$ such that $i r j \in X$, and by $\left(\mathrm{R}_{K}\right)$ an $\langle\phi, j\rangle \in X$. If $j \in W$, then by definition of $R$ in (8.4), $i R j$, and by induction hypothesis $\mathfrak{M}, j \vDash \phi$. So $\mathfrak{M}, i \vDash K \phi$.

Let $\psi:=\langle\neg K \phi, i\rangle \in X \cap\left(\operatorname{FOR}\left(\mathcal{L}_{\mathrm{ARL}}\right) \times W\right)$, for some $i \in \mathbb{N}$. Since $X$ is closed under $\left(\mathrm{R}_{K}\right)$, so for some $j \in \mathbb{N}, \operatorname{irj} \in X$ and $\langle\neg \phi, j\rangle \in X$, since $j$ is new in the set, so $j \in W$. By definition of $R$ in (8.4), $i R j$, and by induction hypothesis, $\mathfrak{M}, j \vDash \neg \phi$. So $\mathfrak{M}, i \vDash \neg K \phi$.

Let $\psi:=\left\langle K^{\diamond} \phi, i\right\rangle \in X \cap\left(\operatorname{FOR}\left(\mathcal{L}_{\mathrm{ARL}}\right) \times W\right)$, for some $i \in \mathbb{N}$. If there is no $i r^{\diamond} j \in X$, then for no $k \in \mathbb{N}, i R^{\diamond} k$, by definition of $R^{\diamond}$ in (8.4), and so $\mathfrak{M}, i \vDash K^{\diamond} \phi$. Let us assume for some $j \in \mathbb{N}$, there is $i r^{\diamond} j \in X$. Since $X$ is closed under rule $\left(\mathrm{R}_{K^{\diamond}}\right)$, so for any $i r^{\diamond} k \in X,\langle\phi, k\rangle \in X$. Then by definition of $R^{\diamond}$ in (8.4), we have $i R^{\diamond} i$. Moreover, since $X$ is closed under rule $\left(\mathrm{R}_{A R L}\right)$, so also $\langle\phi, i\rangle \in X$. But by induction hypothesis $\mathfrak{M}, i \vDash \phi$. Again by definition of $R^{\diamond}$ in (8.4), $\mathfrak{M}, i \vDash K^{\diamond} \phi$.

Let $\left\langle\neg K^{\diamond} \phi, i\right\rangle \in X \cap\left(\operatorname{FOR}\left(\mathcal{L}_{\mathrm{ARL}}\right) \times W\right)$, for some $i \in \mathbb{N}$. Since $X$ is closed under $\left(\mathrm{R}_{\neg K^{\diamond}}\right)$, so for some $j \in \mathbb{N}, \quad i r^{\diamond} j \in X$ and $\langle\neg \phi, j\rangle \in X$. Since $X$ is closed under $\left(\mathrm{R}_{\text {ARL }}\right)$, so $\langle\neg \phi, i\rangle \in X$. However, $i \in W$. So, by induction hypothesis, $\mathfrak{M}, i \vDash \neg \phi$. But, because $i r^{\diamond} j \in X$, so by definition of $R$ in (8.4), $R^{\diamond}(i, i)$. Hence, $\mathfrak{M}, i \vDash \neg K \phi$. 
Finally, we have a theorem on the completeness of tableaux and the proposed semantics for logic $\vdash_{A R L}$.

Theorem 8.7 (Completeness theorem). Let $\models \subseteq P\left(F O R\left(\mathcal{L}_{\text {ARL }}\right)\right) \times F O R\left(\mathcal{L}_{\text {ARL }}\right)$ be the consequence relation defined by the class of models determined by frames $F_{A R L}$. Then for any $X \subseteq \operatorname{FOR}\left(\mathcal{L}_{\mathrm{ARL}}\right), \varphi \in \operatorname{FOR}\left(\mathcal{L}_{\mathrm{ARL}}\right)$ the following facts are equivalent:

1. $X \vDash \varphi$

2. there is a finite subset $Z \subseteq X$ and index $i \in \mathbb{N}$ such that each closure of set $\{\langle\psi, i\rangle: \psi \in Z \cup\{\neg \varphi\}\}$ under set of tableaux rules $\boldsymbol{R}$ is a tableaux inconsistent set of expressions.

Proof. Let us adopt the assumptions. In the theorem proof, we make use of the prior propositions. For implication $(1) \Rightarrow(2)$ lemma (8.6) is sufficient. In turn, for implication $(2) \Rightarrow(1)$ lemma (8.6) is sufficient.

\section{CONCLUSION}

In the paper we proposed a new approach to Fitch Paradox. Our idea was to use single operator of knowability $K^{\diamond}$ instead of composition of $\diamond$ and $K$ in the formulation of Knowability Principle. We treat $K^{\diamond}$ as de re operator, since its interpretation is that Somebody may know that in contrast to It is possible that somebody knows that. We constructed for it a logic in which Fitch paradox disappeared. The logic can be treated as some minimal antirealistic logic, since Knowability Principle is its thesis, while the trivialization of knowledge $(\phi \rightarrow K \phi)$ is not. Of course, other anti-realistic thesis can be added (as $K^{\diamond} \phi \rightarrow \phi$ ), if we want to have a stronger logic. The frames and tableau methods we examined are ready for further development and philosophical discussions.

\section{ACKNOWLEDGMENTS}

The research of Tomasz Jarmużek presented in this article was financed by the National Science Centre, Poland, grant No.: UMO-2015/19/B/ HS1/ 02478 . 


\section{BIBLIOGRAPHY}

Artemov, Sergei, Tudor Protopopescu. 2013. "Discovering knowability: a semantic analysis" Synthese 190, No. 16: 3349-76. DOI: 10.1007/s11229-012-0168-x.

Beal, J.C. 2000. "Fitch's Proof, Verificationism, and the Knower Paradox." Australasian Journal of Philosophy 78, No. 2: 241-47. DOI: 10.1080/00048400012349521.

BEAL, J.C. 2009. "Knowability and Possible Epistemic Oddities." In New Essays on the Knowability Paradox, edited by Joe Salerno, 105-25. Oxford: Oxford University Press. doi: 10.1093/ acprof:oso/9780199285495.003.0009.

VAn Benthem, Johan. 2009. "Actions That Make Us Know.” In New Essays on the Knowability Paradox, edited by Joe Salerno, 129-46. Oxford: Oxford University Press. doi: 10.1093/acprof:oso/9780199285495.003.0010.

VAN BENTHEM, Johan. 2004. "What one may come to know." Analysis 64, No. 282: 95-105. doi: 10.1093/acprof:oso/9780199285495.003.0009

Brogaard, Berit, and Joe Salerno. 2008. "Knowability, Possibility and Paradox." In New Waves in Epistemology, edited by Vincent F. Hendricks and Duncan Pritchard, 270-299. New York: Palgrave Macmillan.

Brogatrd, Berit, and Joe Salerno. 2013. "Fitch's Paradox of Knowability." In The Stanford Encyclopedia of Philosophy, edited by Edward N. Zalta, https://plato.stanford.edu/archives/ win2013/entries/fitch-paradox/, Metaphysics Research Lab, Stanford University, edition: winter 2013.

DeVIdI, David, and Graham Solomon. 2001. "Knowability and Intuitionistic Logic." Philosophia 28, No. 1-4: 319-34.

DuMmetT, Michael. 2001. “Victor's Error.” Analysis 61, No. 1: 1-2. doi: 10.1093/analys/61.1.1.

Fagin, Ronald, Joseph Y. Halpern, Yoram Moses, and Moshe Y. Vardi. 1995. Reasoning about Knowledge. Cambridge, MA: MIT Press.

FISCHER, Martin. 2013. "Some remarks on restricting the knowability principle," Synthese 190, 16: 63-88. DOI 10.1007/s11229-010-9833-0.

Fitch, Frederic B. 2009. “A Logical Analysis of Some Value Concepts.” In New Essays on the Knowability Paradox, edited by Joed Salerno, 21-29. Oxford: Oxford University Press. DOI: 10.1093/acprof:oso/9780199285495.003.0003.

Fuhrmann, André. 2014. "Knowability as potential knowledge." Synthese 191, No. 7: 1627-48. DOI: 10.1007/s11229-013-0340-y.

GoRÉ, Rajeev. 1999. "Tableau systems for modal and temporal logics." In Handbook of tableau methods, edited by Marcello D’Agostino, Dov M. Gabbay, Reiner Hähnle, and Joachim Posegga, 297-396. Dordrecht: Kluwer.

JARMUŻeK, Tomasz. 2013. “Tableau Metatheorem for Modal Logics.” In Trends in Logic. Vol. 41: Recent Trends in Philosphical Logic, edited by Roberto Ciuni, Heinrich Wansing, and Caroline Willkomennen, 103-26. Heidelberg, New York, Dordrecht, London: Springer International Publishing. doi: 10.1007/978-3-319-06080-4_8.

Kennedy, Neil. 2014. "Defending the Possibility of Knowledge." Journal of Philosophical Logic 43, No. 2-3: 579-601. doi: 0.1007/s10992-013-9282-6.

KooI, Barteld. 2016. "The Ambiguity of Knowability." The Review of Symbolic Logic 9, No. 3: 421-28. DOI: 10.1017/S1755020315000416. 
KubYshrina, Ekaterina, and Dmitry V. Zaitsev. 2016. "Rational Agency From a Truth-Functional Perspective." Logic and Logical Philosophy 25, No. 4: 499-520. doi:10.12775/ LLP.2016.016.

Kvanvig, Jonathan. 1995. "The Knowability Paradox and the Prospects for Anti-Realism." Nous 29, No. 4: 481-500. doi: 10.2307/2216283.

Kvanvig, Jonathan. 2006. The Knowability Paradox. Oxford: Oxford University Press.

Maffezioli, Paolo, Alberto NAiBo, and Sara Negri. 2013. "The Church-Fitch knowability paradox in the light of structural proof theory." Synthese 190, No. 14: 2677-716. doi: 10.1007/ s11229-012-0061-7.

MeYer, John Jules Ch. 2001. "Epistemic Logic.” In Blackwell Guide to Philosophical Logic, edited by Lou Gouble. Oxford: Blackwell Publishing.

Meyer, John Jules Ch., and W. van DER Hoek. 1995. Epistemic Logic for AI and Computer Science. Cambridge: Cambridge University Press.

Nelson, Michael. 2019. "The De Re/De Dicto Distinction. Supplement to Propositional Attitude Reports." In The Stanford Encyclopedia of Philosophy, edited by Edward N. Zalta, https://plato.stanford.edu/entries/prop-attitude-reports/dere.html, Metaphysics Research Lab, Stanford University, edition: spring 2019.

Palczewski, Rafał. 2007. "Distributed Knowability and Fitch's Paradox.” Studia Logica 86, No. 3: 455-78. DOI:10.1007/s11225-007-9070-9.

PietruszCZAK, Andrzej, and Tomasz JARMUŻEK. 2018. "Pure modal logic of names and tableau systems.” Studia Logica 106, No. 5: 1261-89. doi: 10.1007/s11225-018-9788-6.

Priest, Graham. 2009. "Beyond the Limits of Knowledge." In New Essays on the Knowability Paradox, edited by Joe Salerno, 93-104. Oxford: Oxford University Press. doi: 10.1093/ acprof:oso/9780199285495.003.0008.

Proietti, Carlo. 2012. “Intuitionistic Epistemic Logic, Kripke Models and Fitch's Paradox.” Journal of Philosophical Logic 41, No. 5: 877-900. doi: 10.1007/s10992-011-9207-1.

Proietti, Carlo. 2016. "The Fitch-Church Paradox and First Order Modal Logic.” Erkenntnis, 81, No. 1: 87-104. doi: 10.1007/s10670-015-9730-5.

SALERnO, Joe. 2009. "Knowability Noir: 1945-1963.” In New Essays on the Knowability Paradox, edited Joe Salerno, 29-48. Oxford: Oxford University Press.

Tennant, Neil, Revamping the Restriction Strategy, in: New Essays on the Knowability Paradox, ed. Salerno J., Oxford University Press, Oxford, 2009, pp. 223-38. doi: 10.1093/acprof:oso/ 9780199285495.003.0015.

Tennant, Neil. 1997. The Taming of the True. Oxford: Oxford University Press.

WANSING, Heinrich. 2002. "Diamonds Are A Philosopher's Best Friends. The Knowability Paradox and Modal Epistemic Relevance Logic." Journal of Philosophical Logic 31: 591-612. doi: 10.1023/A:102125651.

Williamson, Timothy. 1982. "Intuitionism Disproved?" Analysis 42: 203-207. doi: 10.1093/ analys/42.4.203

Williamson, Timothy. 1992. "On Intuitionistic Modal Epistemic Logic.” Journal of Philosophical Logic21: 63-89. doi: 10.1007/BF00126497.

Williamson, Timothy. 2000. Knowledge and Its Limits. Oxford: Oxford University Press.

Zemach, Eddy M. 1987. "Are There Logical Limits For Science?" The British Journal for the Philosophy of Science 38, No. 4: 527-32. doi: 10.1093/bjps/38.4.527. 


\section{KNOWABILITY AS DE RE MODALITY: A CERTAIN SOLUTION TO FITCH PARADOX}

\section{S u m m a r y}

In the paper, we try to find a new, intuitive solution to the Fitch paradox. We claim that traditional expression of Knowability Principle $(p \rightarrow \diamond K p)$ is based on erroneous understanding of knowability as de dicto modality. Instead, we propose to understand knowability as de re modality. In the paper we present the minimal logic of knowability in which Knowability Principle is valid, but Fitch Paradox does not hold anymore. We characterize the logic semantically as well as by an axiomatic and tableaux procedure approach.

Keywords: anti-realism; de dicto modality; de re modality; epistemic logic; Fitch Paradox; knowability logic; knowability principle; modal logic; tableaux methods.

\section{POZNAWALNOŚĆ JAKO MODALNOŚĆ DE RE: PEWNE ROZWIĄZANIE PARADOKSU FITCHA}

\section{Sum mary}

W artykule staramy się znaleźć nowe, intuicyjne rozwiązanie paradoksu Fitcha. Twierdzimy, że tradycyjne wyrażenie zasady poznawalności $(p \rightarrow \diamond K p)$ opiera się na błędnym rozumieniu poznawalności jako modalności de dicto. Zamiast tego proponujemy rozumieć poznawalność jako modalność de re. W artykule przedstawiamy minimalną logikę poznawalności, w której zasada poznawalności jest ważna, ale paradoks Fitcha już nie obowiązuje. Logikę charakteryzujemy semantycznie, a także poprzez podejście aksjomatyczne i tabelaryczne.

Słowa kluczowe: antyrealizm; modalność de dicto; modalność de re; logika epistemiczna; paradoks Fitcha; logika poznawalności; zasada poznawalności; logika modalna; metody tablicowe. 
